\title{
Effect of Dengeling on Bending Fatigue Behaviour of Al Alloy 7050 and Comparison with Milling and Shot Peening
}

\author{
Ru Lin Peng1 ${ }^{, a,{ }^{*}}$, Linnéa Selegård ${ }^{2, b}$, Mattias Jonsson ${ }^{2, c}$, Markus Ess $^{3, d}$ \\ Gert Petersén $^{2, e}$ \\ ${ }^{1}$ Dept of Management and Engineering, Linköping University, SE-581 83 Linköping, Sweden \\ ${ }^{2}$ Saab, S-58188 Linköping, Sweden \\ ${ }^{3}$ Starrag AG, Seebleichestrasse 61, 9404 Rorschacherberg, Switzerland \\ aru.peng@liu.se, ${ }^{b}$ linnea.selegard@saabgroup.com, cmattias.jonsson2@saabgroup.com, \\ dmarkus.ess@starrag.com, "egert.petersen@saabgroup.com
}

Keywords: Dengeling, Shot Peening, Milling, Bending Fatigue, Al Alloy

\begin{abstract}
Dengeling is a new surface mechanical treatment developed as an alternative to the shot peening method used for enhancing the fatigue resistance of metallic materials. In this work, Dengeling is compared with milling and shot peening with regard to the effect on bending fatigue behavior of aluminium alloy AA 7050 T7651. In addition, the influence of certain Dengeling process parameters on the fatigue resistance is studied. Flat bar samples were milled and then subjected to the respective surface treatments. The induced surface integrity changes, namely residual stresses and surface deformation, were characterized by X-Ray diffraction measurement. Four-point bending fatigue tests with a stress ratio of $\mathrm{R}=0.1$ were performed. The results show that all the surface treatments in general improve the fatigue performance of the milled samples but the samples treated by the Dengeling process with similar Almen intensity as the shot peening treatment perform best.
\end{abstract}

\section{Introduction}

Surface mechanical treatments especially shot peening has been developed and widely used by various industries including automative and aerospace to enhance fatigue properties of metallic components [1,2]. The increased fatigue resistance is attributed to beneficial compressive residual stresses and strain hardening induced in a surface layer due to impact of hard shots. Dengeling is a new surface mechanical treatment developed as an alternative method to shot peening. The treatment is carried out by striking the metal surface with a hard indenter to induce surface plastic deformation and compressive residual stresses. Dengeling can be performed on the same machine that is used for machining the component and the operator can control exactly the location and magnitude of the residual stress.

In a previous study [3], it was shown that significant compressive residual stresses can be introduced to a large depth in aluminum alloy 7050 by Dengeling. In the current project, the potential of Dengeling as an effective way to improve the fatigue resistance of milled aluminium alloys was investigated. The fatigue behavior of Dengeling treated AA7050 was studied by the four-point bending fatigue testing and the results were interpreted with respect to the induced changes in residual stresses. Comparison with shot peening was also made.

\section{Experiment details}

Rectangular bars of $10 \mathrm{~mm}$ x $10 \mathrm{~mm}$ x $80 \mathrm{~mm}$ with $1 \mathrm{~mm}$ chamfer on both sides of the surface to be tested in bending fatigue were machined from AA7050 T7651. In addition to the milled samples used as reference, four other groups were treated to different surface conditions, as listed in Table 1. For shot peening (T6) a common process for the alloy was used to compare with a 
Dengeling treatment (T1) of similar Almen intensity as the shot peening. Two other groups (T2 and T3) were selected to investigate the effect of Dengeling process parameters. The dimple overlap is calculated from the percentage of diameter overlapping between two neighboring dimples.

Table 1 Dengeling and shot peening process parameters

\begin{tabular}{|l|l|l|}
\hline Sample group & Treatment & $\begin{array}{l}\text { Indenter size, stroke distance, dimple size, dimple overlap } \\
\text { and line feed direction }\end{array}$ \\
\hline T1 & Dengeling & $\Phi 3 \mathrm{~mm}, 0.2 \mathrm{~mm}, 0.215 \mathrm{~mm}, 25 \%$, parallel \\
\hline T2 & Dengeling & $\Phi 8 \mathrm{~mm}, 0.5 \mathrm{~mm}, 0.72 \mathrm{~mm}, 50 \%$, parallel \\
\hline T3 & Dengeling & $\Phi 8 \mathrm{~mm}, 0.5 \mathrm{~mm}, 0.72 \mathrm{~mm}, 0 \%$, parallel \\
\hline T6 & Shot peening & $\begin{array}{l}\text { Shots: S230H, } \Phi 0.59 \mathrm{~mm} \text {; intensity: } 0.2 \mathrm{mmA} \text {; coverage: } \\
125 \%\end{array}$ \\
\hline M & Milling & \\
\hline
\end{tabular}

The 4-point bending fatigue testing was carried out with a stress ratio of 0.1 and in a frequency of $15 \mathrm{~Hz}$. Samples survived more than $3 \times 10^{6}$ cycles are considered to be runout.

Residual stresses were measured using the X-Ray diffraction technique. The $\mathrm{Cr}-\mathrm{K} \alpha$ radiation was used to measure elastic strains for Al-(311) planes. Upon the assumption of biaxial stress state, in-plane residual stresses were calculated using the $\sin 2 \psi$ method and an elastic constant $1 / 2 \mathrm{~S}_{2}$ of $19.54 \times 10^{-6} / \mathrm{MPa}$. In order to obtain the depth residual stress profile, stepwise layer removal by electrolytic polishing was employed.

Fractographic analysis was performed in SEM to identify the fatigue initiation points and possible origins.

\section{Results and discussion}

Surface morphology. The surface morphology is compared in Fig. 1 between the Dengeling (T1) and shot peening (T6) with similar Almen intensity. As can be seen, a regular pattern of indents was observed for the Dengeling but a random pattern consisting of larger and smaller crats was found for the shot peening. Some small, untreated areas could also be seen for the shot peened surface while full coverage was observed for the Dengeling group. The corresponding roughness is $\mathrm{Ra}=2.3 \pm 0.3 \mu \mathrm{m}$ for $\mathrm{T} 6$ and $\mathrm{Ra}=3.1 \pm 0.8 \mu \mathrm{m}$ for $\mathrm{T} 1$ measured along the line direction and $\mathrm{Ra}=3.1 \pm 0.5 \mu \mathrm{m}$ for measurement along the line feed direction. For Dengeling using the larger indenter (T2 and T3), the surface impression was less obvious and marks from the milling operation were still visible.

Residual stresses. Residual stress profiles for all the sample conditions are presented in Fig. 2. Low residual stresses are observed in the milled sample, as shown in Fig. 2a. As expected, the shot peening process introduced a compression layer of about $0.3 \mathrm{~mm}$ with maximum compressive stress slightly over $300 \mathrm{MPa}$ and significant plastic deformation as indicated by the diffraction peak broadening (Fig. 2b). Comparison between Fig. $2 b$ and c reveals that the corresponding Dengeling process generated a larger compression layer (about $0.4 \mathrm{~mm}$ ), lower surface plastic deformation and similar maximum subsurface compressive residual stress in the axial direction while the transverse compressive stresses were much larger.

For the two Dengeling treatments using the large indenter (Fig. 2d and e) the compression layer thickness was about doubled as compared to shot peening, the maximum compressive stresses and surface deformation are however smaller than for both shot peening and Dengeling with the small indenter. The lowest surface deformation and compressive stresses were found with the treatment with no dimple overlap (T3). 

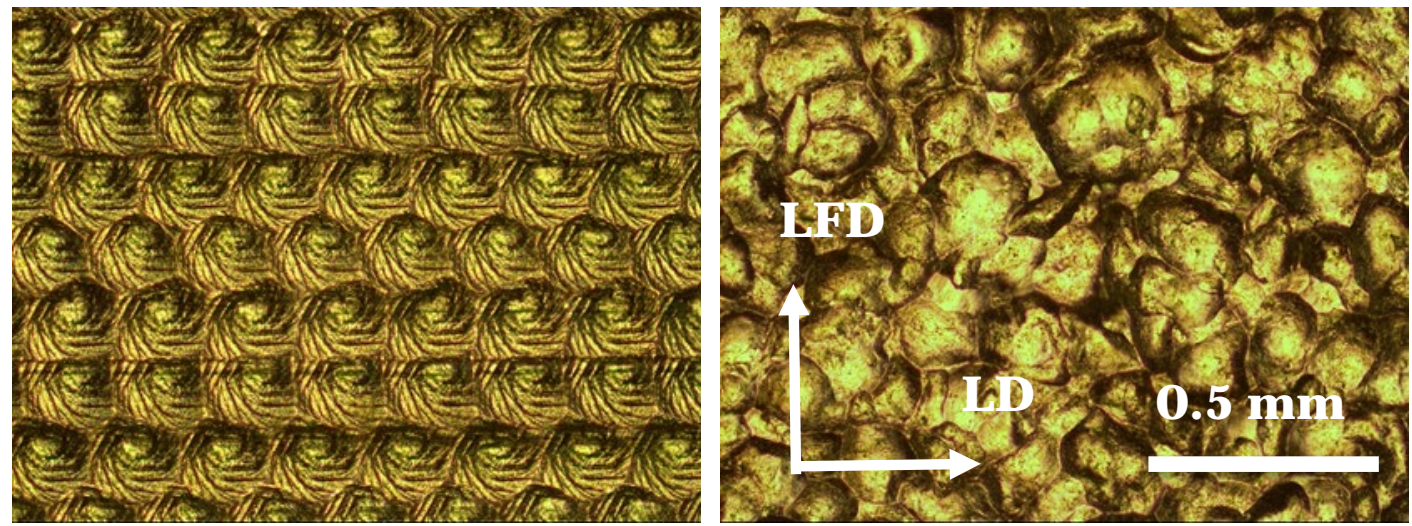

Figure 1. Micrograph showing surface indents on the shot peened (left) and Dengeling sample (T1) (right). LD: line direction; LFD: line feed direction.
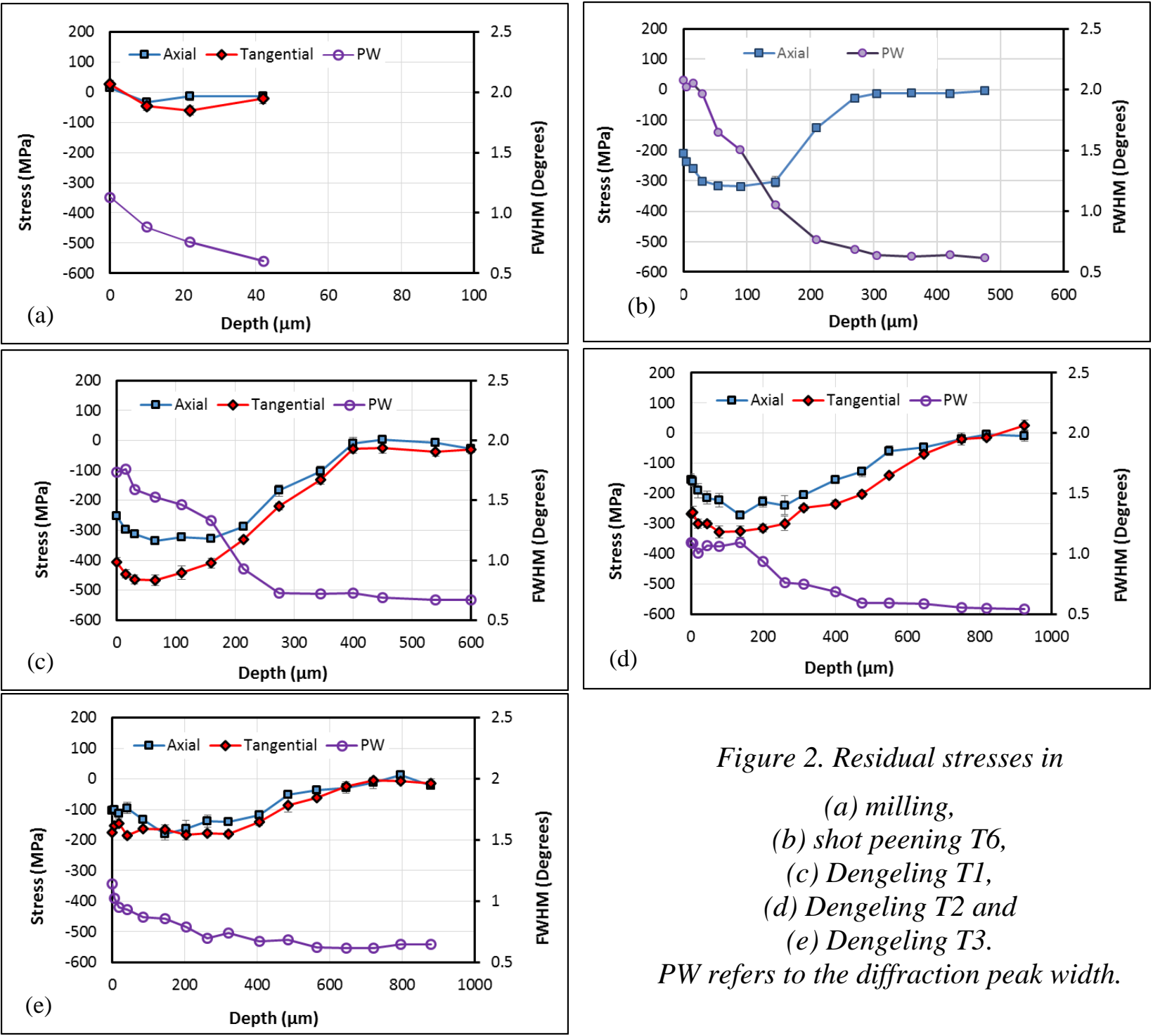

Figure 2. Residual stresses in

(a) milling,

(b) shot peening $T 6$,

(c) Dengeling T1,

(d) Dengeling T2 and

(e) Dengeling T3.

$P W$ refers to the diffraction peak width.

Fatigue. The fatigue testing results are presented as S-N plots in Fig. 3 and 4. On the assumption of elastic loading, the maximum stress in the surface was calculated according to the 
bending beam theory. XRD measurements were made on Dengeling treated and shot peened samples that fractured from a fatigue load of $436 \mathrm{MPa}$. The obtained residual stresses were similar to the as treated samples, indicating no global yielding in the surface of the samples during fatigue loading at and below $436 \mathrm{MPa}$.
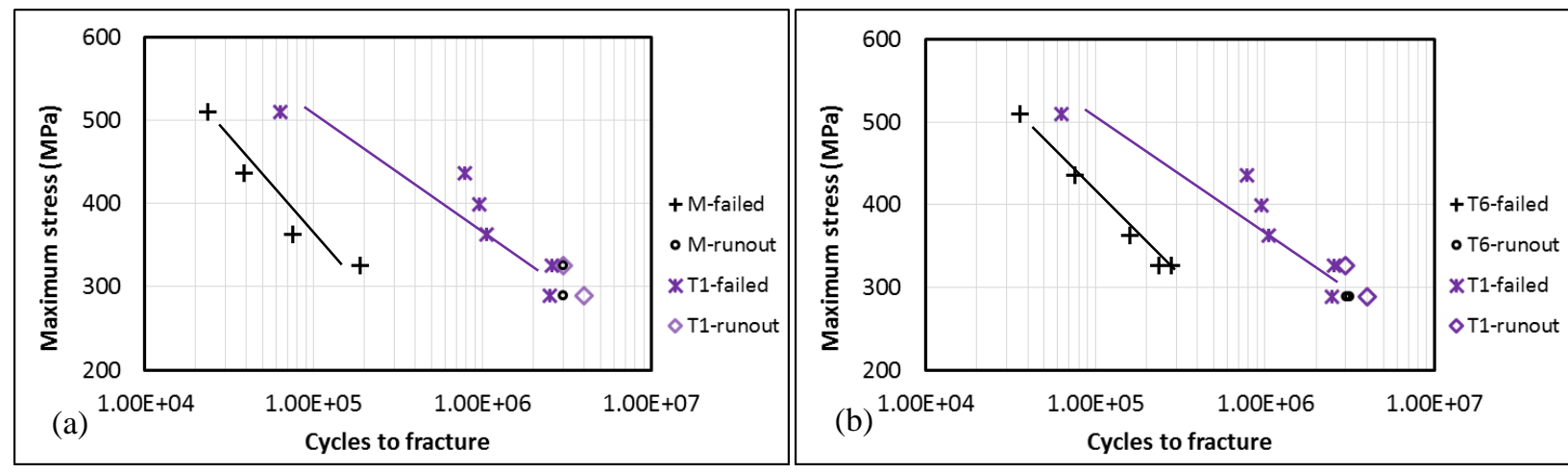

Figure 3 Comparison of Dengeling (T1) with milling (M) (a) and with shot peening (SP) (b). The lines serve as a guide to the eye.
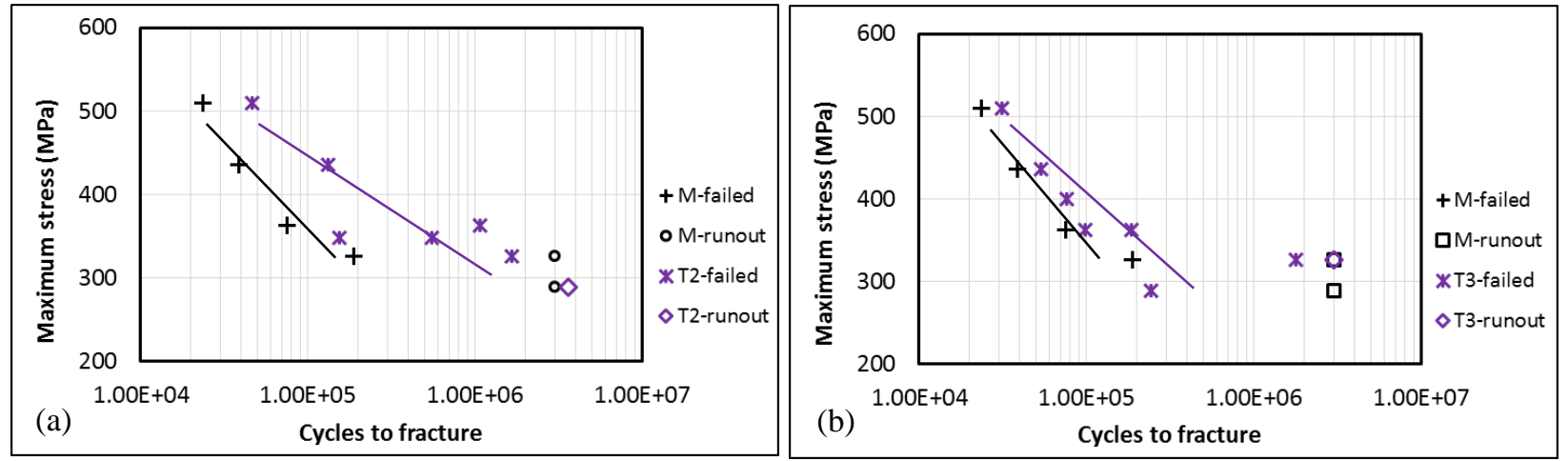

Figure 4. Comparison of Dengeling treatment using $\Phi 8 \mathrm{~mm}$ indenter and $50 \%$ dimple overlapping (T2) and 0\% overlapping (T3) with milling (M). The lines serve as a guide to the eye.

The Dengeling group (T1) is compared with the milling group (M) in Fig. 3a. As can be seen, application of the Dengeling treatment greatly improved the fatigue performance of milled samples. More than one order of magnitude better in fatigue life was observed for the slope region. At the lower stress, $326 \mathrm{MPa}$, both runout and failure were observed. Nonetheless, the failed Dengeling sample had a much longer life than the milled sample. Comparison for the lowest stress level is difficult as only one milled sample was tested.

An increase of fatigue life up to $100 \%$ in the slope region was obtained by the shot peening. The improvement is however much less in comparison with the Dengeling treatment as Fig. 3b illustrates. At the lowest stress level, the two treatments seem to show similar behavior. One Dengeling sample and two shot peened samples survived 3 million loading cycles while the Dengeling samples failed with a fatigue life close to 3 million cycles. Actually the two "Runout" samples of shot peening failed shortly after continued loading beyond 3 million cycles.

The fatigue life of T2 samples in Fig. 4a was twice to three times of the milled samples in the upper part of the slope region. However, in the lower part of the slope region the fatigue data scattered largely, from about $1.55 \times 10^{5}$ to over $3 \times 10^{6}$ cycles. The T3 group presented in Fig. $4 \mathrm{~b}$ 
was treated with same parameters as T2 but with no dimple overlap. For this group, the improvement in fatigue life was marginal in the slope region and varied in the low stress region.

Fractography. Fractographic analysis reveals that surface crack initiation, preferably at precipitates, and its propagation resulted in fatigue failure of the milled samples. For the Dengeling samples, the fatigue damage always started below the surface and the initiation site moved closer to the surface with increasing applied stress. For loading at and below $363 \mathrm{MPa}$, the crack initiation site was located about 500 to $560 \mu \mathrm{m}$ below surface, i.e. outside the compression zone which is about $400 \mu \mathrm{m}$. For larger applied stresses, the fatigue cracking started inside the compression zone. It can be concluded that the Dengeling treatment successfully suppressed the surface crack initiation. Examples of fracture surface revealing the crack initiation sites (indicated by arrows) are given in Fig. 5a for milling and Fig. 5b for Dengeling treated samples.
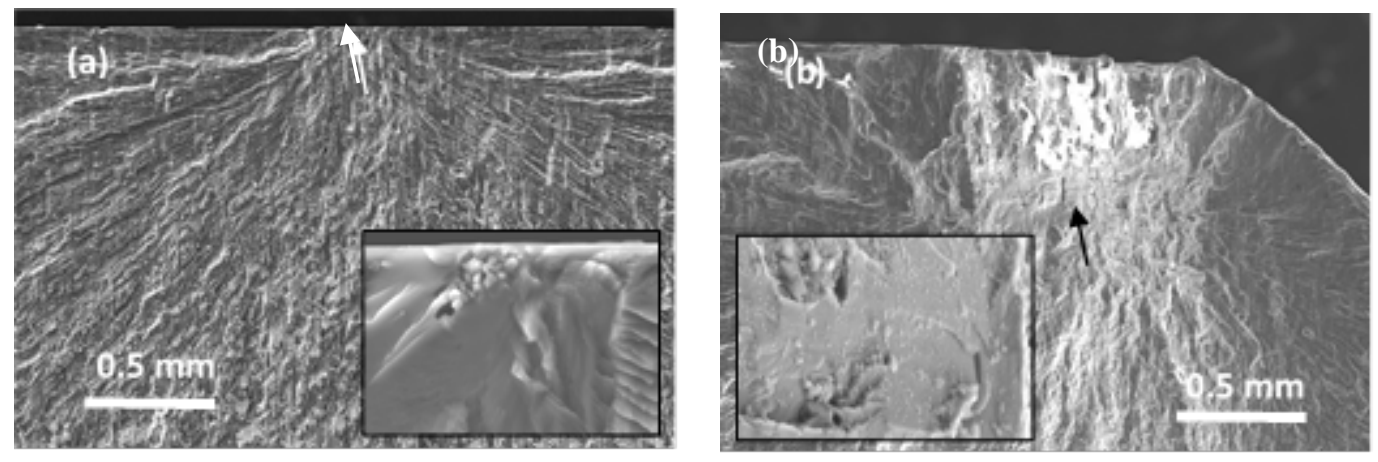

Figure 5. Crack initiation from (a) the surface of an M sample that failed at $363 \mathrm{MPa}$ and (b) subsurface (about $560 \mu \mathrm{m}$ below surface) of a T1 sample that failed under $326 \mathrm{MPa}$. The insets reveal the respective crack initiation site.
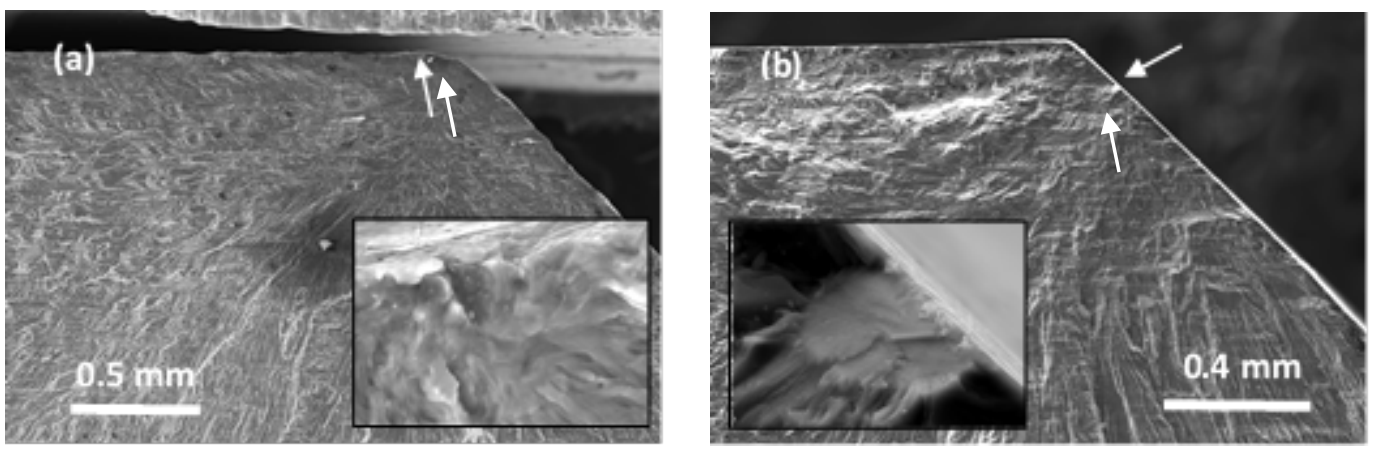

Figure 6 Surface crack initiation in (a) a T6 sample failed at 326 MPa and (b) a T2 sample failed at $363 \mathrm{MPa}$. The insets show the respective fatigue initiation site at the surface.

In spite of the surface compressive residual stresses, fatigue cracks tend to start from surface or very near to the surface, see Fig. 6a, in the shot peened group. In another word, the shot peening process employed is less effective in suppressing surface crack initiation in comparison with the Dengeling treatment.

For the T2 samples, fatigue crack origins were observed mostly on the chamfer surface, see Fig. 6b, although crack initiation near the edge of the top flat surface was also observed. The treatment of the chamfer surfaces was made separately from the top flat surface. The chamfer surfaces are more prone to fatigue, which means that the strengthening was not as effective as on the top flat surface. It could be due to the indenter diameter that is much larger than the width of 
the chamfer surface. Similar to the milled group, surface crack initiation often resulted in final failure in T3 samples. The $0 \%$ overlap means that about $21.5 \%$ of the surface was not covered by the indents. Such bared areas could become preferable sites for crack initiation.

\section{Concluding remarks}

The effect of a surface mechanical treatment on fatigue originates from the induced changes in surface integrity especially surface roughness, strain hardening and compressive residual stresses [1]. The results in the previous section reveal that for the Dengeling and shot peening treatments using similar Almen intensity, the magnitude of compressive residual stresses in the fatigue loading direction is similar and better surface roughness with a higher degree of strain hardening was found in the shot peened samples. However, the Dengeling treatment is much more effective for fatigue resistance reinforcement in the slope region of the S-N graph. This is likely attributed to that the Dengeling treatment successfully suppressed surface initiation of fatigue cracks where the shot peening failed. For the shot peened samples subjected to higher loading stresses, microcracks might exist or quickly develop from other types of surface defects and the positive effect of shot peening can be explained by the retardation of crack growth by the compressive residual stresses. For the Dengeling samples in the same stress region, fatigue cracks initiated below the surface, which is a much slower process and the more significant improvement may come from the delay of fatigue crack initiation. At the low stress region, surface crack initiation may become difficult for the shot peened samples and therefore the behavior of both groups are similar. It should be pointed out that the fatigue crack initiation sites tend to be located close to the edge near the chamfer especially for the shot peened samples.

Dengeling treatments using the large indenter generated a much deeper compression zone. However, as fatigue crack initiates often from the weaker chamfer surface in T2 or untreated surface areas in T3, both treatments are less effective and the fatigue data are scattered in the low stress region.

Large intermetallic particles are common in the alloy. Those located at or near surface may result in microcracks during shot peening or Dengeling. EDS analysis of crack initiation sites also reveals such precipitates at crack origins in a number of failed samples in all conditions: milling, shot peening and Dengeling. These particles and their distribution could be responsible for scattered data near the runout stress region.

\section{Acknowledgement}

This project is carried out within the Strategic Innovation Program "Metallic Materials", a joint venture of Vinnova, Formas and Energy Agency of Sweden.

\section{Reference}

[1] K. A. Soady, "Life assessment methodologies incoroporating shot peening process effects: Mechanistic consideration of residual stresses and strain hardening: Part 1 - Effect of shot peening on fatigue resistance,” Mater. Sci. Technol. (United Kingdom), vol. 29, no. 6, pp. 637651, 2013. https://doi.org/10.1179/1743284713Y.0000000222

[2] A. Azhari, S. Sulaiman, and A. K. P. Rao, "A review on the application of peening processes for surface treatment," in IOP Conference Series: Materials Science and Engineering, 2016, vol. 114, no. 1. https://doi.org/10.1088/1757-899X/114/1/012002

[3] L. Selegård, R. Lin Peng, A. Billenius, G. Petersén, M. Ess, M. Jonsson, "Residual Stresses in Dengeling-Treated Aluminum Alloy AA 7050," in Residual Stresses 2016: ICRS-10, 2016, pp. 425-430. 